\section{Working memory and interference: A comment on Jenkins, Myerson, Hale, and Fry (1999)}

\author{
KLAUS OBERAUER \\ University of Potsdam, Potsdam, Germany \\ and \\ HEINZ-MARTIN SÜß \\ University of Mannheim, Mannheim, Germany
}

\begin{abstract}
Jenkins, Myerson, Hale, and Fry (1999) showed that slopes relating complex spans to simple spans were considerably smaller than one, indicating that persons with higher simple spans suffered more interference when the span lask was combined with a processing demand. They argued that this finding ruled out accounts of working memory based on interference and/or inhibition of interfering information. We demonstrate that the effect is mainly an artifact from regression to the mean, owing to the low reliability of span scores as used by Jenkins et al. Data from 133 young adults for two verbal and two spatial span tasks show that the slopes relating complex to simple performance are considerably higher for sum scores than for span scores. Furthermore, an adequate test for an interference or an inhibition account of working memory is to predict interference from complex span tasks, not from simple span tasks. Interference effects in the verbal span tasks were negatively correlated with an independent measure of working memory capacity, consistent with the interference/inhibition account.
\end{abstract}

In a recent article, Jenkins, Myerson, Hale, and Fry (1999) presented results that, on their interpretation, question the validity of inhibition-based accounts of working memory capacity. Basically, a meta-analysis of data from their laboratory showed that the difference between simple spans (i.e., memory spans without secondary processing demand) and complex spans (i.e., spans with an additional processing demand) was larger in people with higher simple spans. This was reflected in slopes substantially less than one for the regression of complex spans on simple spans. Jenkins et al. interpreted the difference between simple and complex spans as an interference effect resulting from the secondary task. Notably, the inverse relationship between simple span and the interference effect held only within age groups. Differences in simple span between age groups were not

The research reported in this paper was supported by Grant Wi 1390/1-2 from the Deutsche Forschungsgemeinschaft. We thank Randall Engle, Michael Humphreys, Joel Myerson, and Reinhold Kliegl for comments on previous versions of the manuseript. Correspondence concerning this article should be addressed to K. Oberauer, Allgemeine Psychologie I, University of Fotsdam, P. O. Box 60 15 53, 14415 Potsdam, Germany (e-mail: ko@rz.uni-potsdam.de). accompanied by systematic variation in the interference effect.

Jenkins et al. (1999) argued that this finding is incompatible with the idea that individual differences in working memory capacity (at least in age-homogeneous samples) are related to differences in susceptibility to interference and/or the capacity to inhibit irrelevant information. Accounts based on interference and inhibition, they argued, should predict the opposite pattern: smaller, not larger, interference effects in people with higher spans. As an alternative, they presented an account based on decay from working memory. Assuming a negatively accelerated decay function, the absolute amount of decay over a constant time interval is larger for a high-span person than for a low-span person. If high- and low-span persons do not differ systematically in the speed with which they perform the secondary task (i.e., span and speed are uncorrelated), high spans are expected to suffer a larger interference effect than do low spans.

We disagree with this interpretation for both methodological and conceptual reasons. On the methodological side, we will argue that the slopes smaller than one are most likely due to a statistical artifact, regression to the mean. On the conceptual side, we will argue that simple and complex spans do not measure the same construct and that an inhibition account of working memory predicts that people with higher complex spans show less interference but not necessarily that people with higher simple spans show less interference. To illustrate our point, we will present data that replicate the original findings from Jenkins et al. (1999) but, in addition, allow more thorough analyses that point toward an alternative explanation. Our comment focuses on the issue of interference and inhibition as a potential factor driving individual differences in working memory; it does not challenge the conclusions regarding age differences drawn by Jenkins et al. We do not wish to defend a particular theory that assumes that the ability to inhibit interfering information underlies individual differences in working memory capacity, but only to point out that the data presented by Jenkins et al. do not justify the rejection of such an account.

\section{RELIABILITY AND REGRESSION TO THE MEAN}

The regression line relating two variables is crucially dependent on the variable's reliability (Cohen \& Cohen, 1983). In the extreme case, in which one variable has zero reliability (i.e., consists exclusively of error variance), the correlation between predictor and criterion is zero, and the regression line will be flat. More generally, everything else being equal, the slope of the regression 
line decreases with decreasing reliability of the variables involved.

The span tasks used in the meta-analysis were scored with the Wechsler digit span procedure, in which tasks of increasing length are presented and the experimenter discontinues testing whenever a participant fails to recall correctly both lists of a given length. This procedure yields only a single value for each participant, so no direct estimates of reliability are available. Jenkins et al. (1999) briefly discuss this problem and conclude that measurement error does not compromise their conclusions, because the reliability of the traditional WAIS-R Digit Span is known to be above 90 .

We think that this is a highly questionable generalization. The reliability estimates in the Wechsler intelligence test manual are based on a representative sample from the general population. The studies summarized in Jenkins et al. (1999) recruited mainly undergraduates for the young adult groups; this is most likely associated with a restriction of range, thereby reducing the ratio of true variance to total variance. Moreover, the span tasks used by Jenkins et al. differed from the WAIS-R Digit Span in several ways that could make a difference for their reliability. For example, their presentation times per item differed from those used in the WAIS-R. Perhaps more important, their complex spans introduced an additional processing demand. This could turn the task into one qualitatively different from the simple span as measured in the WAIS-R; it might even measure a different construct (Daneman \& Carpenter, 1980; Engle, Tuholski, Laughlin, \& Conway, 1999). This concern is supported by the fact that the correlations between simple and complex spans were only .42 (for the two verbal tasks) and .33 (for the two spatial tasks). Given that simple and complex spans are highly similar and, therefore, should share most of their task-specific variance, these correlations are suspiciously low. This could indicate that at least one of the two spans is not as reliable as is assumed, or alternatively, that the two variables do not measure the same construct. Either way, the assumption that both spans have reliabilities of at least .90 seems not well grounded.

Since we have no knowledge about the reliability of the span tasks involved in Jenkins et al.'s (1999) metaanalysis, it is difficult to assess to what degree the regression slopes smaller than one are an artifact of measurement error. Therefore, we decided to replicate their kind of analysis on a data set of our own with simple and complex span tasks for which reliability estimates are available.

\section{METHOD}

\section{Participants}

The participants were 133 students from the University of Mannheim, who took part on 2 days of testing involving various intelligence and working memory tests. Their mean age was 25.8 years $(S D=3.8)$, and $44 \%$ were female

\section{Materials and Procedure}

We analyzed data from two simple spans (one verbal and one spatial) and their corresponding complex counterparts. All the tasks were computer administered.

Word span task. A list of frequent German nouns (none longer than 12 characters) was displayed one by one, each word for $1 \mathrm{sec}$, in the center of the screen. Immediately after the last word, the participants attempted to write down the words in correct order on an answer sheet containing the appropriate number of slots for each list length. Following two practice trials, three trials for each list length between four and eight words were presented in ascending order of list length.

Dual word span task. The dual-task version differed from the the simple word span task in two ways. First, the memory list was presented simultaneously for $1 \mathrm{sec}$ times the number of words in the list. Second, the presentation of the memory list was followed by a fixed time interval, during which the participants had to perform a choice reaction time task. This task involved classifying words as referring to an animal or a plant by pressing one of two keys on the computer keyboard. New stimuli were presented immediately after each reaction until a total of $5 \mathrm{sec}$ has elapsed. The participants were instructed to react as quickly and accurately as possible in order to accomplish as many reaction time tasks as possible in the 5-sec interval. After two practice trials, three trials of each list length between three and seven words were administered in ascending order.

Pattern span task. A sequence of partially filled $3 \times 3$ grids was presented. Each pattern was shown for $3 \mathrm{sec}$. The series was reproduced immediately after the last pattern by marking the corresponding cells in empty matrices on the answer sheet. There were five trials each for the list lengths of three to five, presented in ascending order.

Dual pattern span task. The dual-task version was identical to the simple pattern span, except for an additional processing demand, which consisted of classifying arrows as pointing upward or downward. The processing task was again administered for a constant interval of $5 \mathrm{sec}$. There were five trials for each list length from two to four.

In both cases, the simple span task immediately preceded the corresponding dual-task version. Furthermore, the choice reaction time tasks involved in the dual-task versions were administered as single tasks about half an hour before, so the participants had a moderate amount of practice with both components when they encountered the dual-task versions.

Two additional tasks relevant in the present context were computeradministered versions of the reading span task (Daneman \& Carpenter, 1980) and its numerical analogue, the computation span task (Turner \& Engle, 1989). The reading span task began with the presentation of a number of short sentences, one by one, for 4 sec each. The participants were required to evaluate the sentences as true or false by pressing an appropriate key and to remember the last word of each sentence. The last words had to be written down in correct order after the last sentence of an item was presented. There were 11 trials, with list length ranging from four to seven. The computation span task used simple equations, involving addition or subtraction of single digits, instead of sentences. The results of the equations had to be recalled in correct order. There were 11 trials, with list lengths varying from five to eight.

\section{RESULTS}

For each task, a sum score was computed as follows: For each trial, the number of words or patterns reproduced correctly at the correct ordinal position was counted, and these numbers were added over all the trials. To compare 
simple and complex spans on the sum measure, we used only the trials with list lengths administered in both versions (i.e., list lengths from four to seven for word span tasks and from three to four for pattern span tasks). This means that the list length of eight (word span), list length of three (dual word span), list length of five (pattern span), and list length of two (dual pattern span) were not included in the sum scores.

As a second score, we simulated a span procedure with the data from the two word span tasks: For each list length, we selected the first two of the three trials and scored whether a participant succeeded in recalling perfectly both lists, one list, or none of them. The third trial of each list length was ignored in order to make our span score parallel the span procedure of Jenkins et al. (1999) as much as possible. Starting with the shortest list length (i.e., four for the single and three for the dual word spans), a participant's span was increased by one for each list length for which he or she recalled at least one of the two lists perfectly. This was done for increasing list lengths, up to the shortest list length on which the participant failed on both trials. At this point, the scoring procedure was stopped and all items from longer lists were ignored, because two successive failures on a given list length means that testing is discontinued in the usual span procedure. The participant was credited with the score reached up to this point (which equaled the list length of the longest list passed at least once before the first double failure). If only one of the two trials from the previous list length was recalled correctly, span was decreased by 0.5 . Those participants who failed on the two shortest lists were assigned a span equal to the shortest list length minus one.

No span score was formed for pattern span, because there were not enough different list lengths. Table 1 summarizes the means and standard deviations for both scores. In addition, we estimated the reliability of the sum scores by Cronbach's alpha, which corresponds to the mean of all possible split-half reliability values of the items entering into the score. No such reliability estimation was possible for the span scores, because they were not defined as linear combinations of independent items. Table 2 presents the correlations of the six variables.

Following the procedure of Jenkins et al. (1999), we also conducted all the analyses after exclusion of those participants whose spans could be underestimated because they never reached a list length on which they failed twice. The largest list length tested was eight for

Table 1

Mean Sum and Span Scores (with Standard Deviations) and Reliability Estimates for Simple and Complex Spans

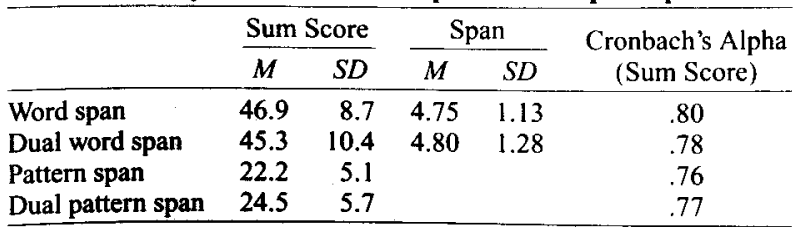

simple word span and seven for dual word span, so we excluded participants with simple word spans larger than seven and those with complex word spans larger than six. This reduced the sample size to 110 . The analyses with the reduced sample showed signs of a moderate restriction of range but did not alter any of the conclusions. We therefore decided to present mainly the results from the unrestricted sample and to add data from the reduced sample in parentheses.

Several important observations can be made from these data. First, complex span scores were not systematically lower than the simple span scores, which is different from what Jenkins et al. (1999) found. This could mean that there was not much interference at all in the complex span tasks. Alternatively, the interference that did occur was outweighed by the moderate amount of practice with the memory tasks that the participants already had when they first attacked the dual-task versions. In any case, we will see that the absolute amount of interference is not crucial to our argument.

A second phenomenon to notice is the surprisingly low correlation between the sum scores and the span scores from the same tasks. These two scores are supposed to measure the same variable. In addition, the correlations between simple and complex word span tasks were much higher for the sum scores (.72) than for the span scores (.46). Note that the latter value is comparable with the task intercorrelations reported by Jenkins et al. (1999). Both findings together lead us to suspect that the span scores had low reliability. We cannot assess their reliability directly, but if we assume that sum scores and span scores measure the same variable, there are two ways to do so indirectly.

An observed correlation $r_{\mathrm{obs}}(x, y)$ can be expressed as a function of the true correlation $r_{\text {true }}(x, y)$ and the reliabilities of the variables involved (Cohen \& Cohen, 1983, Equation 2.11.4):

$$
r_{\text {obs }}(x, y)=r_{\text {true }}(x, y)<r(x, x) r(y, y) .
$$

Let $r(x, x)$ denote the reliability of a sum score and $r(y, y)$ the reliability of the corresponding span score. Equation 1 can now be solved for the unknown reliability $r(y, y)$ :

$$
r(y, y)=\frac{r_{\text {obs }}(x, y)^{2}}{r_{\text {true }}(x, y)^{2} r(x, x)} .
$$

If sum scores $(x)$ and span scores $(y)$ measure the same variable, their true correlation is one. Hence, we can insert 1 for $r_{\text {true }}(x, y)$ and Cronbach's alpha values from Table 1 for $r(x, x)$ to compute an approximation for $r(y, y)$ from Equation 2. This yields reliability estimates for the span scores of simple word span of .72 and for complex (dual) word span of .74.

A second way to estimate the span scores' reliability is to compute the correlation between the sum scores for simple and complex tasks and correct for attenuation ac- 
Table 2

Intercorrelations of Sum Scores and Spans

\begin{tabular}{lccccc}
\hline & $\begin{array}{c}\text { Dual Word Span } \\
\text { (Sum) }\end{array}$ & $\begin{array}{c}\text { Pattern Span } \\
\text { (Sum) }\end{array}$ & $\begin{array}{c}\text { Dual Pattern Span } \\
\text { (Sum) }\end{array}$ & $\begin{array}{c}\text { Word Span } \\
\text { (Span) }\end{array}$ & $\begin{array}{c}\text { Dual Word Span } \\
(\text { Span) }\end{array}$ \\
\hline Word span (sum) & .72 & .23 & .17 & $.76(.72)$ & $.60(.51)$ \\
Dual word span (sum) & & .27 & .26 & $.56(.48)$ & $.76(.66)$ \\
Pattern span (sum) & & & .67 & $.17(-.04)$ & $.15(-.01)$ \\
Dual pattern span (sum) & & & & $.17(.08)$ & $.12(.01)$ \\
Word span (span) & & & & $.46(.34)$ \\
\hline
\end{tabular}

Note-Correlations $>.15$ are significant at $p<.05$. Values in parentheses refer to a reduced sample $(N=110)$, from which the participants with simple spans $>7$ or complex spans $>6$ were excluded.

cording to Equation 1. This means solving Equation 1 for $r_{\text {true }}(x, y)$, which is taken to represent the true correlation between the sum scores of simple and complex spans. With the reliabilities for the two variables (see Table 1), we can estimate their true correlation from the observed correlation. This yields an estimate for the true correlation between simple and complex word spans of $r=.91$. The same true correlation should hold for the span scores of simple and complex word spans, because they are assumed to measure the same two constructs. From this, we can obtain an estimation for the joint reliability of the two span scores, $r(x, x) r(y, y)$, by transforming Equation 2:

$$
r(y, y) r(x, x)=\frac{r_{\text {obs }}(x, y)^{2}}{r_{\text {true }}(x, y)^{2}} .
$$

If we assume for simplicity that the two span scores have equal reliability, this reliability equals the square root of $r(x, x) r(y, y)$, which is .51. The bottom line is that the span scores must be assumed to have a reliability of between .51 and .74 , considerably lower than that of the sum scores.

Next, we estimated the regression line for the prediction of complex spans from simple spans. The results are summarized in Table 3. The upper panel of Figure 1 shows the scatterplot for the sum scores of word span as an illustrative example.

As can be seen, the slopes were considerably lower than one in all three cases, thus reproducing the findings of Jenkins et al. (1999). It is also clear, however, that the slope was much lower for the span scores than for the sum scores of word span. This is a direct consequence of the lower task intercorrelation for the span scores and, most likely, a result of the span scores' lower reliability. It might seem surprising that we could reproduce the pat-

Table 3

Regressions of Dual Tasks on Simple Tasks

\begin{tabular}{lccc}
\hline & Slope & Intercept & $R^{2}$ \\
\hline Word span (sum) & 0.85 & 5.28 & 0.51 \\
Pattern span (sum) & 0.74 & 7.96 & 0.44 \\
Word span (span) & $0.53(0.40)$ & $2.30(2.62)$ & $0.22(0.12)$
\end{tabular}

Note-Values in parentheses refer to a reduced sample $(N=110)$, from which the participants with simple spans $>7$ or complex spans $>6$ were excluded. tern found by Jenkins et al. even though there was no mean effect of interference in our data: How can there be more interference in high-span individuals than in lowspan individuals if there is no evidence of interference overall? The answer is that the phenomenon observed by Jenkins et al.- shallow slopes relating complex to simple spans-does not necessarily reflect the amount of interference suffered by individuals. The shallow slopes are simply a by-product of the comparatively low correlation between simple and complex spans. This low correlation is at least in part due to the unreliability of the span measures.

The powerful effect of regression to the mean can be illustrated by reversing the $x$ - and $y$-axes of the regression lines. If Jenkins et al. (1999) were right in claiming that low-span individuals experience less interference than do high-span individuals, the slopes of simple span plotted over complex span should be larger than one. Following the line of argument proposed by Jenkins et al., the interference cost in this kind of plot would be the deviation of the regression line above the main diagonal (i.e., the advantage of single task scores above dual task scores). The lower panel of Figure 1 shows such a reversed plot for the sum scores of the two word span tasks. As can be seen, the slope is again smaller than one. Taken together, the two analyses visualized in Figure 1 show that regression to the mean can produce slopes considerably smaller than one, regardless of which variable is used as the predictor and which as the criterion!

\section{SHORT-TERM MEMORY AND WORKING MEMORY}

We believe, however, that reliability and regression to the mean is not the full story. Even with perfect reliability, simple and complex span tasks will not be perfectly correlated. This brings us to our second, conceptual argument. There is good evidence in the literature that simple and complex memory span tasks do not measure the same construct (Cantor, Engle, \& Hamilton, 1991; Daneman \& Carpenter, 1980; Daneman \& Merikle, 1996; Engle et al., 1999; Oberauer, 1993). Simple spans and complex spans tend to load on different factors and correlate with different external criteria. In particular, simple spans are notoriously bad predictors of complex reasoning, whereas complex spans predict reasoning ability 

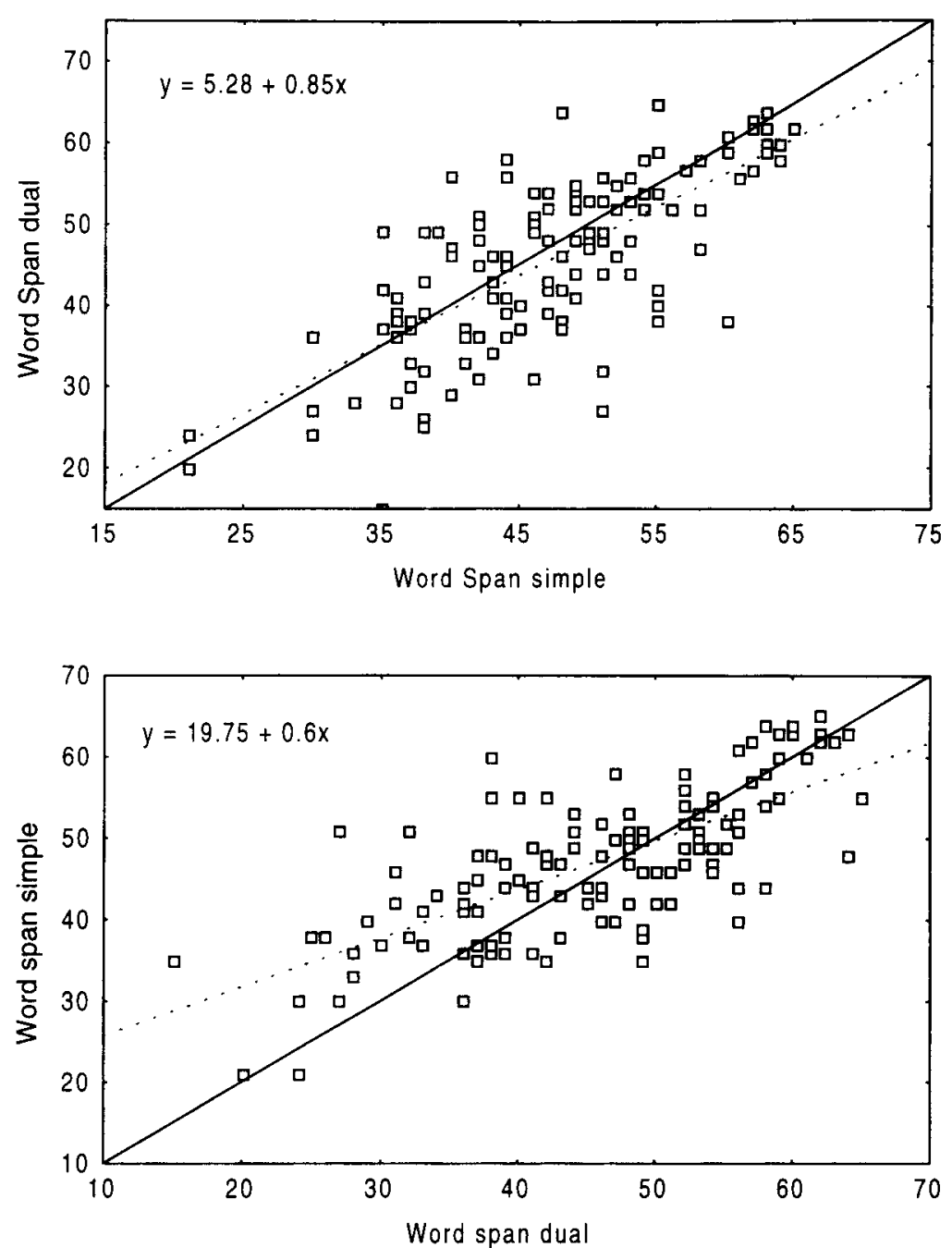

Figure 1. Scatterplots of dual word span over simple word span, sum scores (top panel), and simple word span over dual word span, sum scores (bottom panel). The broken line represents the estimated regression line; the continuous line represents the main diagonal (slope $=1) . N=133$.

well. This led investigators to the assumption that complex span tasks, but not simple span tasks, measure working memory capacity (e.g., Daneman \& Carpenter, 1980). Jenkins et al. (1999) explicitly disagreed with this position, stating that "simple word span tasks also exemplify the use of working memory" (p. 28) according to Baddeley (1986). This is certainly true if we consider the whole working memory system as envisioned by Baddeley, including the phonological loop. Researchers drawing a distinction between simple and complex span measures, however, maintain that simple spans reflect mainly the capacity of a peripheral system, such as the phonological loop, whereas complex spans reflect the capacity of the central component of working memory to a much larger degree (e.g., Cantor et al., 1991). Therefore, from Baddeley's framework, one would predict that simple and complex spans are not perfectly correlated- although both reflect an aspect of working memoryand this is in full accordance with the facts.

Theories of working memory that assign interference and inhibition a central role for individual differences are concerned mainly with the central executive, not with the slave systems in Baddeley's (1986) model (e.g., Engle, Kane, \& Tuholski, 1999). This implies that individual differences in complex span, but not necessarily individual differences in simple span, reflect individual differences in susceptibility to interference and the capacity to inhibit irrelevant information. And of course, it is only the complex span, not the simple span variant, in which the issue of inhibiting irrelevant information arises at all, because only the complex spans require that the representations used in the secondary tasks be suppressed quickly when no longer necessary. It follows that an interference/inhibition account of working memory 


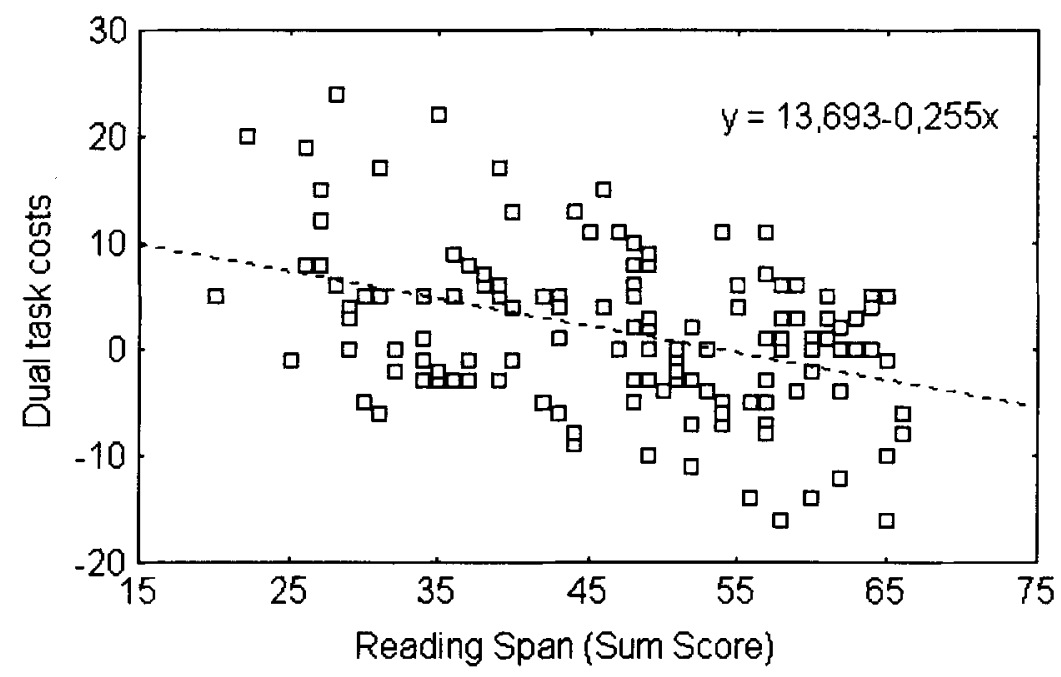

Figure 2. Interference costs (difference of simple and dual word span sum scores) as a function of reading span. $N=133$.

capacity does predict that individuals with higher complex spans will experience less interference than people with lower complex spans. But it does not predict that persons with higher simple spans will experience less interference.

This implies that the slope of the regression line with simple span tasks as predictors and complex tasks as criteria is not an adequate test for an inhibition-based account of working memory. The complex span tasks, but not the simple span tasks, can be regarded as good measures of the central executive's capacity. Therefore, a working memory model based on inhibition or interference makes the straightforward prediction that individuals with high complex span will experience less interference than will individuals with low complex span. In terms of regression analysis, this means that the regression slope for simple span as a function of complex span will be smaller than one (and the intercept will be positive). This is so because the interference effect, measured as the difference between simple and complex spans, is predicted to decrease with higher complex spans. Unfortunately, the same artifact of regression to the mean that led Jenkins et al. (1999) to reject the inhibition hypothesis will work in favor of the hypothesis in this analysis (for an illustration, see Figure 1). One way to test the inhibition hypothesis could be to measure complex span and the interference effect separately. The minimal design necessary for this test includes one pair of measures for simple and complex span tasks and an additional, independent measure of complex span. The first two tasks can be used to obtain the simple-to-complex difference; the second complex task can be used to predict this difference. An interference/inhibition account should predict a negative covariation between the difference score and the independent estimate of complex span.
Fortunately, there were two additional complex span tasks available in our study that could be used as independent predictors for the interference effects in the word span and the pattern span tasks. We built sum scores for reading span and computation span and correlated them with the differences between the simple and the complex versions of the two other span tasks (the differences were computed from the sum scores). The difference of simple and complex word spans correlated -.41 with reading span and -.33 with computation span; both correlations were highly significant. Figure 2 plots the simple-to-complex differences as a function of reading span. The difference of simple and complex pattern spans correlated -.05 and .01 with the two predictors, which was not significant. Thus, the predicted negative correlation between complex span and the interference effect was obtained when all the tasks used material from the verbal-numerical domain. There was no evidence for a relationship between predictors involving verbalnumerical materials and the interference effect computed from spatial tasks. This difference could be a hint that the ability to resist interference is domain specific. But as long as it is not replicated with a different set of tasks, it could as well be accidental and should, therefore, be interpreted cautiously. The important point is that there was no positive association between the interference effect and an independent measure of complex span.

\section{DISCUSSION}

The data and analyses presented above show several important points. The slope of the regression line for complex span scores as a function of simple span scores was consistently smaller than one, in agreement with the findings from Jenkins et al. (1999). When the same data 
were analyzed using sum scores, the slopes were much higher, but still smaller than one.

The substantial difference between sum scores and span scores is most likely due to the low reliability of the latter. Although the span's reliability cannot be estimated directly from the present data, we obtained converging indirect evidence that the span scores have low reliability. This evidence involves the relatively low correlation of span and sum scores computed from the same raw data and the comparatively low correlation between simple and complex span scores. One reason why the span scores had lower reliability than the sum scores is that the former included only two items for each list length, as in the Jenkins et al. (1999) studies, whereas the latter included three. A more general reason why span scores have low reliability could be that the scoring procedure throws away information. First, each item is only scored as passed or failed; the number of list elements recalled correctly is not measured. Second, after a participant fails twice on a given list length, his or her performance on longer lists is no longer considered.

We conclude that the findings of Jenkins et al. (1999) do not rule out a working memory model based on interference and inhibition. The positive covariation between simple span and the "interference effect" they observed is, in large part, an artifact from regression to the mean, owing to low reliability of the span scores. The remaining true positive covariation is not relevant for a working memory theory based on interference and inhibition, because interference is only predicted to be low for people with high scores on complex span tasks. An unbiased test of this prediction requires that the difference between simple and complex span scores-that is, the interference effect-be measured independently from the complex span score that is used as its predictor. Preliminary evidence from our data is consistent with the prediction of the interference/inhibition account. More systematic research, however, is required to test the generality of this finding--in particular, in a constellation of tasks that elicit larger mean interference costs.

\section{REFERENCES}

BADDELEY, A. D. (1986). Working memory. Oxford: Oxford University Press, Clarendon Press.

Cantor, J., Engle, R. W., \& Hamilton, G. (1991). Short-term memory, working memory, and verbal abilities: How do they relate? Intelligence, 15, 229-246.

COHEN, J., \& COHEN, P. (1983). Applied multiple regression/correlation analysis for the behavioral sciences ( $2 \mathrm{nd} \mathrm{ed}$.). Hillsdale, NJ: Erlbaum.

Daneman, M., \& Carpenter, P. A. (1980). Individual differences in working memory and reading. Journal of Verbal Learning \& Verbal Behavior, 19, 450-466.

Daneman, M., \& Merikle, P. M. (1996). Working memory and language comprehension: A meta-analysis. Psychonomic Bulletin \& Review, 3, 422-433.

Engle, R. W., Kane, M. J., \& Tuholski, S. W. (1999). Individual differences in working memory capacity and what they tell us about controlled attention, general fluid intelligence, and functions of the prefrontal cortex. In A. Miyake \& P. Shah (Eds.), Models of working memory: Mechanisms of active maintenance and executive control (pp. 102-134). Cambridge: Cambridge University Press.

Engle, R. W., Tuholski, S. W., Laughlin, J. E., \& Conway, A. R. A. (1999). Working memory, short term memory and general fluid intelligence: A latent variable approach. Journal of Experimental Psychology: General, 128, 309-331.

Jenkins, L., Myerson, J., Hale, S., \& Fry, A. F. (1999). Individual and developmental differences in working memory across the life span. Psychonomic Bulletin \& Review, 6, 28-40.

Oberauer, K. (1993). Die Koordination kognitiver Operationen: Eine Studie zum Zusammenhang von "working memory" und Intelligenz [The coordination of cognitive operations: A study on the relationship of working memory and intelligence]. Zeitschrift für Psychologie, 201, 57-81.

TURNER, M. L., \& ENGLE, R. W. (1989). Is working memory capacity task dependent? Journal of Memory \& Language, 28, 127-154.

(Manuscript received June 22, 1999; revision accepted for publication November 10, 1999.) 